\title{
Edition and translation
}

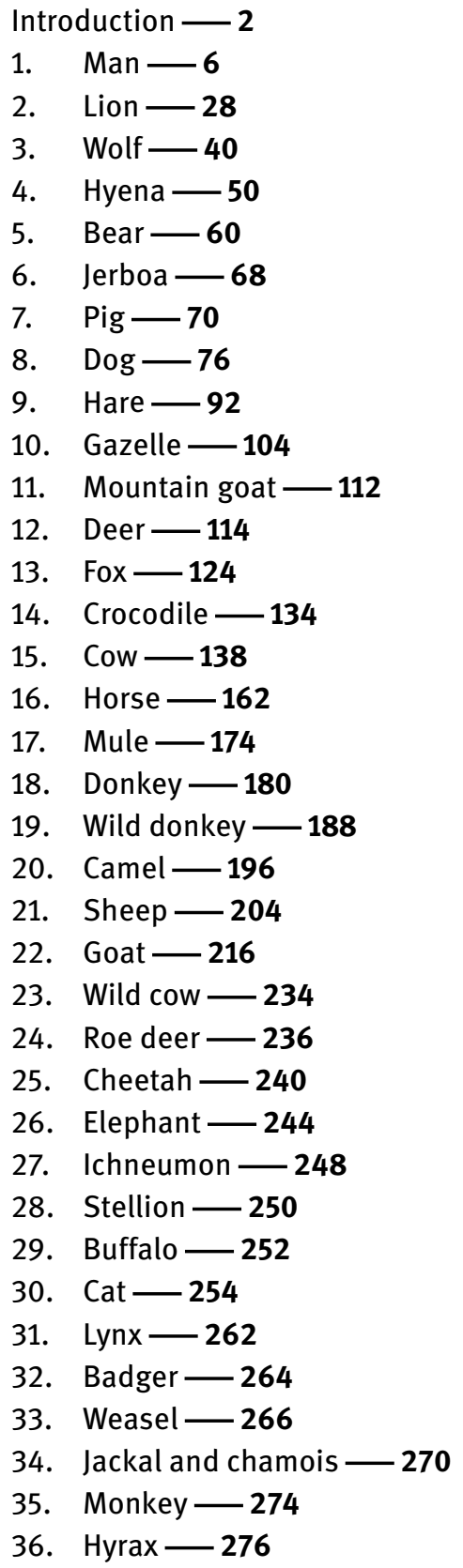
37. Beaver $-\mathbf{2 7 8}$
38. Hedgehog $-\mathbf{2 8 0}$
39. Indian hedgehog - 288
40. Kāša $\mathbf{2 9 0}$
41. Salamander -292
42. Rhinoceros -294
43. Mouse -296
44. Mole - 302
45. Sandgrouse $-\mathbf{3 0 8}$
46. Vulture - 312
47. Collared pigeon -316
48. Wood pigeon -318
49. Hoopoe $-\mathbf{3 2 0}$
50. Swallow - 328
51. Quail - 336
52. Sparrow -338
53. Bat -344
54. Abū tamra bird -356
55. Pigeon -358
56. Egyptian vulture -364
57. Starling - $\mathbf{3 7 0}$
58. Kite - 372
59. Crow -376
60. Owl - 386
61. Rooster 392
62. Hen - 398
63. Ostrich -404
64. Goose and duck -406
65. Crane $-\mathbf{4 1 2}$
66. Bustard -416
67. Saker falcon $-\mathbf{4 1 8}$
68. Peacock $-\mathbf{4 2 0}$
69. Curlew -422
70. Eagle -424
71. Zummağ falcon $-\mathbf{4 2 8}$
72. Sparrow hawk -430
73. Merlin -432

74. Hawk - 434

75. Green woodpecker - $\mathbf{4 3 6}$

76. Guinea fowl -438

77. Turtledove $-\mathbf{4 4 0}$

78. Wild pigeon -444

79. Snake - 448

80. Scorpion - 458

81. Ladybird -464

82. Lizard - 466

83. Dung beetle -472

84. Chameleon -474

85. Blister beetle $-\mathbf{4 7 8}$

86. White worm $-\mathbf{4 8 0}$

87. Gecko - 482

88. Fly -486

89. Spanish fly $-\mathbf{4 8 8}$

90. Hornet $-\mathbf{4 9 0}$

91. Mantis $-\mathbf{4 9 2}$

92. Ant -494

93. Scarab -498

94. Bedbug $-\mathbf{5 0 2}$

95. Lice - $\mathbf{5 0 4}$

96. Flea - $\mathbf{5 0 6}$

97. Spider $-\mathbf{5 0 8}$

98. Kanğ $-\mathbf{5 1 0}$

99. Earthworm $-\mathbf{5 1 4}$

100. Sperm whale - $\mathbf{5 1 6}$

101. River crab - $\mathbf{5 1 8}$

102. Sea crab $-\mathbf{5 2 2}$

103. Whale $-\mathbf{5 2 4}$

104. Turtle $-\mathbf{5 2 6}$

105. River snail $-\mathbf{5 3 4}$

106. Pearl oyster -536

107. Leech $-\mathbf{5 4 0}$

108. Frog -544

109. Freshwater fish $\mathbf{-} \mathbf{5 5 0}$

110. Saltwater fish $-\mathbf{5 5 2}$ 
Appendix - Synoptic Table of the Manuscript Tradition — 561

Thematic Index 573

Arabic-English glossary - $\mathbf{5 7 6}$

1. Animal parts and substances $-\mathbf{5 7 6}$

2. Other simples $-\mathbf{5 7 7}$

3. Diseases $-\mathbf{5 8 1}$

English Arabic glossary - 584

1. Animal parts and substances $-\mathbf{5 8 4}$

2. Other simples $-\mathbf{5 8 5}$

3. Diseases $\mathbf{5 8 9}$ 
